\title{
Induced Graphoidal Decompositions in Product Graphs
}

\author{
Mayamma Joseph $^{1}$ and I. Sahul Hamid ${ }^{2}$ \\ ${ }^{1}$ Department of Mathematics, Christ University, Bangalore, Karnataka 560029, India \\ ${ }^{2}$ Department of Mathematics, The Madura College, Madurai, Tamil Nadu 625011, India
}

Correspondence should be addressed to I. Sahul Hamid; sahulmat@yahoo.co.in

Received 25 July 2012; Revised 20 October 2012; Accepted 5 November 2012

Academic Editor: Annalisa De Bonis

Copyright (C) 2013 M. Joseph and I. S. Hamid. This is an open access article distributed under the Creative Commons Attribution License, which permits unrestricted use, distribution, and reproduction in any medium, provided the original work is properly cited.

\begin{abstract}
Let $G$ be a nontrivial, simple, finite, connected, and undirected graph. A graphoidal decomposition (GD) of $G$ is a collection $\psi$ of nontrivial paths and cycles in $G$ that are internally disjoint such that every edge of $G$ lies in exactly one member of $\psi$. By restricting the members of a GD $\psi$ to be induced, the concept of induced graphoidal decomposition (IGD) of a graph has been defined. The minimum cardinality of an IGD of a graph $G$ is called the induced graphoidal decomposition number and is denoted by $\eta_{i}(G)$. An IGD of $G$ without any cycles is called an induced acyclic graphoidal decomposition (IAGD) of $G$, and the minimum cardinality of an IAGD of $G$ is called the induced acyclic graphoidal decomposition number of $G$, denoted by $\eta_{i a}(G)$. In this paper we determine the value of $\eta_{i}(G)$ and $\eta_{i a}(G)$ when $G$ is a product graph, the factors being paths/cycles.
\end{abstract}

\section{Introduction}

By a graph $G=(V, E)$ we mean a nontrivial, finite, connected, and undirected graph having no loops and multiple edges. The order and size of graph $G$ are denoted by $n$ and $m$ respectively. For terms not defined here we refer the reader to [1].

A decomposition of $G$ is a collection $\psi=\left(H_{1}, H_{2}\right.$, $H_{3}, \ldots, H_{k}$ ) of edge-disjoint subgraphs of $G$ such that every edge of $G$ belongs to exactly one $H_{i}$. Graph decomposition problems constitute a major area of research because of their theoretical and practical implications. Designing interconnection networks and drug designing are examples for the application of graph decomposition problems. Among the variants of decompositions of graphs that abound in the literature, path decomposition problems assume a prominent position. Harary [2] introduced the concept of path decomposition of graphs in 1970 which was further studied by Harary and Schwenk [3], Péroche [4], and Stanton et al. [5]. As a special case of path decomposition, Acharya and Sampathkumar [6] introduced the notion of graphoidal decomposition which is a decomposition of a graph into internally disjoint paths/cycles. By imposing the condition that the members of a graphoidal decomposition are induced paths/cycles, Arumugam [7] introduced the concept of induced graphoidal decomposition as well as that of induced acyclic graphoidal decomposition. Studies on these decompositions were initiated by Ratan Singh and Das $[8,9]$ and were further extended by Sahul Hamid and Joseph $[10,11]$ by obtaining certain bounds of the related parameters $\eta_{i}$ and $\eta_{i a}$ and solving some characterization problems. In this paper we determine the value of $\eta_{i}$ and $\eta_{i a}$ for a class of product graphs, namely, products of paths and cycles.

\section{Induced Graphoidal Decomposition}

The concept of graphoidal cover (we say graphoidal decomposition) introduced by Acharya and Sampathkumar [6] is defined as follows.

Definition 1 (see [6]). A graphoidal decomposition (GD) of a graph $G$ is a collection $\psi$ of non-trivial paths and cycles of $G$ such that

(i) every vertex of $G$ is an internal vertex of at most one member of $\psi$,

(ii) every edge of $G$ is in exactly one member of $\psi$. 
The minimum cardinality of a GD of a graph $G$ is called the graphoidal decomposition number of $G$ and is denoted by $\eta(G)$.

Note that if $P=\left(v_{0}, v_{1}, \ldots, v_{k}\right)$ is a path, not necessarily open, in a graph $G$, then $v_{0}$ and $v_{k}$ are called terminal vertices and $v_{1}, v_{2}, \ldots, v_{k-1}$ are called internal vertices of $P$. For cycles (considered as closed paths), there is an inherent "ordering" of vertices as in paths. So, when we say that a cycle $C$ of a graph $G$ is a member of a GD $\psi$ of $G$, we should mention the vertex at which the cycle $C$ begins, and this particular vertex is considered as the terminal vertex of $C$ and all other vertices on $C$ are called internal vertices of $C$. Given a GD $\psi$ of $G$, a vertex $v$ is said to be interior to $\psi$ if $v$ is an internal vertex of an element of $\psi$ and is called exterior to $\psi$ otherwise.

As a variation of GD, Arumugam [7] formulated the concept of induced graphoidal decomposition by restricting the members of a GD to be induced paths/cycles.

Definition 2 (see [7]). An induced graphoidal decomposition (IGD) of a graph $G$ is a GD $\psi$ of $G$ in which every member of $\psi$ is an induced path/cycle. The minimum cardinality of an IGD of $G$ is called the induced graphoidal decomposition number of $G$ and is denoted by $\eta_{i}(G)$ or $\eta_{i}$.

Arumugam [7] further defined an induced acyclic graphoidal decomposition (IAGD) of a graph $G$ as a GD of $G$ in which every member is an induced path. The minimum cardinality of an IAGD of $G$ is called the induced acyclic graphoidal decomposition number of $G$ and is denoted by $\eta_{i a}(G)$ or $\eta_{i a}$.

The study of the parameters $\eta_{i}$ and $\eta_{i a}$ was initiated by Ratan Singh and Das $[8,9]$ by determining the values of these path-decomposition parameters for families of graphs such as complete graphs, complete bipartite graphs, unicyclic graphs, and bicyclic graphs. Sahul Hamid and Joseph $[10,11]$ extended this study further and obtained bounds for $\eta_{i}(G)$ as well as $\eta_{i a}(G)$ in terms of the diameter, girth, and maximum degree of a vertex of $G$ and also characterized graphs obtaining these bounds.

The following theorem which gives the value of $\eta_{i}$ in terms of the size $m$ of a graph $G$ and the maximum number of interior vertices of an IGD of $G$ is useful in determining the value of $\eta_{i}$ for a given graph.

Theorem 3 (see [10]). For every IGD $\psi$ of a graph $G$, let $t_{\psi}$ denote the number of vertices interior to $\psi$ and let $t_{i}(G)=$ $\max t_{\psi}$, where the maximum is taken over all the IGD $\psi$ of G. Then $\eta_{i}(G)=m-t_{i}(G)$.

Remark 4. If there exists an edge-disjoint collection $\delta$ of internally disjoint induced paths/cycles of $G$ such that each vertex of $G$ is an internal vertex of some element of $\mathcal{S}$, then $\eta_{i}(G)=m-n$ and vice versa. This is because $\mathcal{S}$ together with the edges of $G$ not belonging to members of $\mathcal{S}$ yields an IGD $\psi$ of $G$ with $t_{i}(G)=n$.

We require the following theorem giving the value of $\eta_{i}$ for complete graphs.
Theorem 5 (see [8]). For the complete graph $K_{n}$ one has

$$
\eta_{i}\left(K_{n}\right)= \begin{cases}1, & \text { if } n=3, \\ 4, & \text { if } n=4, \\ m-n, & \text { if } n \geq 6, n \text { is even, } \\ m-n+1, & \text { if } n \geq 5, n \text { is odd. }\end{cases}
$$

The following result is analogous to Theorem 3 and is useful in determining $\eta_{i a}(G)$ for a given graph $G$.

Theorem 6 (see [11]). For any graph $G, \eta_{i a}(G)=m-t_{i a}(G)$, where $_{i a}(G)$ denotes the maximum number of vertices of $G$ that can be made interior to an IAGD of G.

Remark 7. $\eta_{i a}(G)=m-n$ if and only if there exists an edgedisjoint collection $\delta$ of internally disjoint induced paths of $G$ such that each vertex of $G$ is an internal vertex of some path in $\delta$.

\section{Induced Graphoidal Decomposition Number of Products of Paths and Cycles}

Given two graphs $G$ and $H$, their product is a graph whose vertex set is $V(G) \times V(H)=\{(g, h): g \in V(G)$ and $h \epsilon$ $V(H)\}$ while the edge set varies according to the nature of the product. Various kinds of graph products are defined in [12]. We consider only the Cartesian product, strong product, and lexicographic product of two paths/cycles. The Cartesian product of graphs $G$ and $H$ denoted by $G \square H$ has the edge set $E(G \square H)=\left\{\left((g, h),\left(g^{\prime}, h^{\prime}\right)\right): g=g^{\prime},\left(h, h^{\prime}\right) \in E(H)\right.$ or $\left.\left(g, g^{\prime}\right) \in E(G), h=h^{\prime}\right\}$, and the strong product of graphs $G$ and $H$ is denoted by $G \otimes H$ with edge set $E(G \otimes H)=$ $\left\{\left((g, h),\left(g^{\prime}, h^{\prime}\right)\right): g=g^{\prime},\left(h, h^{\prime}\right) \in E(H) ;\left(g, g^{\prime}\right) \in E(G), h=\right.$ $h^{\prime}$ or $\left(g, g^{\prime}\right) \in E(G)$ and $\left.\left(h, h^{\prime}\right) \in E(H)\right\}$. The lexicographic product or the composition of graphs $G$ and $H$ is denoted by $G \circ H$ where $E(G \circ H)=\left\{\left((g, h),\left(g^{\prime}, h^{\prime}\right)\right):\left(g, g^{\prime}\right) \in\right.$ $E(G)$ or $\left.g=g^{\prime},\left(h, h^{\prime}\right) \in E(H)\right\}$.

All through this section and the next, we use the following notations. For positive integers $n_{1}$ and $n_{2}$,

$$
\begin{aligned}
& P_{n_{1}}=\left(u_{1}, u_{2}, u_{3}, \ldots, u_{n_{1}}\right), \\
& P_{n_{2}}=\left(v_{1}, v_{2}, v_{3}, \ldots, v_{n_{2}}\right), \\
& C_{n_{1}}=\left(u_{1}, u_{2}, u_{3}, \ldots, u_{n_{1}}, u_{1}\right), \\
& C_{n_{2}}=\left(v_{1}, v_{2}, v_{3}, \ldots, v_{n_{2}}, v_{1}\right) .
\end{aligned}
$$

For all the product graphs $G$ we have considered, the vertex set $V(G)=\left\{w_{i j}: 1 \leq i \leq n_{1} ; 1 \leq j \leq n_{2}\right\}$, where $w_{i j}=\left(u_{i}, v_{j}\right)$.

First we determine the value of $\eta_{i}$ for the Cartesian products of two paths/cycles.

Theorem 8. For the graph $G=P_{n_{1}} \square P_{n_{2}}$, where $n_{1}, n_{2} \geq 2$, one has

$$
\eta_{i}(G)= \begin{cases}1, & \text { if } n_{1}=n_{2}=2, \\ m-n+2, & \text { if } n_{1}=2, n_{2} \geq 3, \\ m-n, & \text { otherwise. }\end{cases}
$$


Proof. We have $E(G)=\left\{w_{i j} w_{i(j+1)}: 1 \leq i \leq n_{1} ; 1 \leq j \leq\right.$ $\left.\left(n_{2}-1\right)\right\} \cup\left\{w_{i j} w_{(i+1) j}: 1 \leq i \leq\left(n_{1}-1\right) ; 1 \leq j \leq n_{2}\right\}$.

If $n_{1}=n_{2}=2$, then $G=C_{4}$ and obviously $\eta_{i}(G)=$ 1. When $n_{1}=2$ and $n_{2} \geq 3$, let $Q_{1}=\left(w_{21}, w_{11}, w_{12}\right.$, $\left.w_{13}, \ldots, w_{1 n_{2}}, w_{2 n_{2}}\right), Q_{2}=\left(w_{21}, w_{22}, w_{23}, \ldots, w_{2 n_{2}}\right)$ and $S^{\prime}=$ $\left\{w_{1 j} w_{2 j}: 2 \leq j \leq\left(n_{2}-1\right)\right\}$. Then $Q_{1}$ and $Q_{2}$ along with the set $S^{\prime}$ form an IGD $\psi$ of $G$ with cardinality $n_{2}$ so that $\eta_{i}(G) \leq|\psi|=n_{2}=m-n+2$. Also for any IGD $\psi$ of $G$, at least one vertex of each of the cycles $\left(w_{11}, w_{21}, w_{22}, w_{12}, w_{11}\right)$ and $\left(w_{1 n_{2}}, w_{1\left(n_{2}-1\right)}, w_{2\left(n_{2}-1\right)}, w_{2 n_{2}}, w_{1 n_{2}}\right)$ will be exterior and therefore $t_{i}(G) \leq n-2$. Hence by Theorem 3 we get $\eta_{i}(G) \geq$ $m-n+2$ proving that $\eta_{i}(G)=m-n+2$.

Now, suppose $n_{1}, n_{2} \geq 3$. Then consider the following paths and cycles:

$$
\begin{aligned}
Q^{\prime}= & \left(w_{22}, w_{21}, w_{11}, w_{12}, w_{22}\right), \\
Q_{1}= & \left(w_{12}, w_{13}, \ldots, w_{1 n_{2}}, w_{2 n_{2}}, \ldots, w_{n_{1} n_{2}}, w_{n_{1}\left(n_{2}-1\right)}, \ldots,\right. \\
& \left.w_{n_{1} 1}, w_{\left(n_{1}-1\right) 1} \ldots, w_{21}\right), \\
Q_{2}= & \left(w_{n_{1} 2}, w_{\left(n_{1}-1\right) 2}, \ldots, w_{32}, w_{22}, w_{23}, \ldots, w_{2 n_{2}}\right), \\
Q_{i}= & \left(w_{i 2}, w_{i 3}, \ldots, w_{i n_{2}}\right) \text { for each } i=3,4,5, \ldots,\left(n_{1}-1\right) .
\end{aligned}
$$

Then $\mathcal{S}=\left\{Q^{\prime}, Q_{1}, Q_{2}, \ldots, Q_{\left(n_{1}-1\right)}\right\}$ is an edge-disjoint collection of internally disjoint induced paths and cycles of $G$ having all the vertices of $G$ as internal vertices of members of $\mathcal{S}$. Therefore by Remark 4 , we have $\eta_{i}(G)=m-n$, thus proving the theorem.

Theorem 9. If $G=C_{n_{1}} \square C_{n_{2}}$, where $n_{1}, n_{2} \geq 3$, then $\eta_{i}(G)=$ $m-n$.

Proof. The edge set $E(G)=\left\{w_{i j} w_{i(j+1)}: 1 \leq i \leq n_{1} ; 1 \leq j \leq\right.$ $\left.\left(n_{2}-1\right)\right\} \cup\left\{w_{i n_{2}} w_{i 1}: 1 \leq i \leq n_{1}\right\} \cup\left\{w_{i j} w_{(i+1) j}: 1 \leq i \leq\right.$ $\left.\left(n_{1}-1\right) ; 1 \leq j \leq n_{2}\right\} \cup\left\{w_{n_{1} j} w_{1 j}: 1 \leq j \leq n_{2}\right\}$. Now, let

$$
\begin{aligned}
Q_{1} & =\left(w_{11}, w_{12}, w_{13}, \ldots, w_{1 n_{2}}, w_{11}\right), \\
Q_{2} & =\left(w_{1 n_{2}}, w_{2 n_{2}}, w_{3 n_{2}}, \ldots, w_{n_{1} n_{2}}, w_{1 n_{2}}\right), \\
Q_{3} & =\left(w_{n_{1} n_{2}}, w_{n_{1}\left(n_{2}-1\right)}, w_{n_{1}\left(n_{2}-2\right)}, \ldots, w_{n_{1} 1}, w_{n_{1} n_{2}}\right), \\
Q_{4} & =\left(w_{n_{1} 1}, w_{\left(n_{1}-1\right) 1}, w_{\left(n_{1}-2\right) 1}, \ldots, w_{11}, w_{n_{1} 1}\right), \\
Q_{5} & =\left(w_{n_{1} 2}, w_{\left(n_{1}-1\right) 2}, \ldots, w_{32}, w_{22}, w_{23}, \ldots, w_{2 n_{2}}\right), \\
Q_{(i-2)}^{\prime} & =\left(w_{i 2}, w_{i 3}, \ldots, w_{i n_{2}}\right) \text { for each } i=3,4, \ldots,\left(n_{1}-1\right) .
\end{aligned}
$$

Then $\mathcal{S}=\left\{Q_{1}, Q_{2}, \ldots, Q_{5}, Q_{1}^{\prime}, Q_{2}^{\prime}, \ldots, Q_{\left(n_{1}-3\right)}^{\prime}\right\}$ is a collection of induced paths and cycles that are edge-disjoint as well as internally disjoint where each vertex of $G$ is internal to a path/cycle of $\mathcal{S}$. Hence it follows from Remark 4 that $\eta_{i}(G)=$ $m-n$.

Theorem 10. For the graph $G=P_{n_{1}} \square C_{n_{2}}$, where $n_{1} \geq 2, n_{2} \geq$ $3, \eta_{i}(G)=m-n$.
Proof. We have $E(G)=\left\{w_{i j} w_{i(j+1)}: 1 \leq i \leq n_{1} ; 1 \leq j \leq\right.$ $\left.\left(n_{2}-1\right)\right\} \cup\left\{w_{i n_{2}} w_{i 1}: 1 \leq i \leq n_{1}\right\} \cup\left\{w_{i j} w_{(i+1) j}: 1 \leq i \leq\right.$ $\left.\left(n_{1}-1\right) ; 1 \leq j \leq n_{2}\right\}$. Let

$$
\begin{aligned}
& Q_{1}=\left(w_{12}, w_{11}, w_{21}, w_{2 n_{2}}\right), \\
& Q_{2}=\left(w_{21}, w_{22}, w_{12}, w_{13}, \ldots, w_{1 n_{2}}\right), \\
& Q_{3}=\left(w_{22}, w_{23}, w_{24}, \ldots, w_{2 n_{2}}, w_{1 n_{2}}, w_{11}\right), \\
& Q^{\prime}=\left(w_{2 n_{2}}, w_{3 n_{2}}, \ldots, w_{n_{1} n_{2}}, w_{n_{1} 1}\right), \\
& Q_{j}^{\prime \prime}=\left(w_{2 j}, w_{3 j}, w_{4 j}, \ldots, w_{n_{1} j}, w_{n_{1}(j+1)}\right), \\
& j=1,2, \ldots\left(n_{2}-1\right) .
\end{aligned}
$$

Then by taking $\mathcal{S}=\left\{Q_{1}, Q_{2}, Q_{3}\right\}$ when $n_{1}=2$ and $\mathcal{S}=$ $\left\{Q_{1}, Q_{2}, Q_{3}, Q^{\prime}, Q_{1}^{\prime \prime}, Q_{2}^{\prime \prime}, \ldots, Q_{\left(n_{2}-1\right)}^{\prime \prime}\right\}$ for $n_{1} \geq 3$ we get the collection of internally disjoint induced paths satisfying the property mentioned in Remark 4 and therefore $\eta_{i}(G)=m-$ $n$.

Next we proceed to obtain the value of $\eta_{i}$ for the strong product of two paths/cycles.

Theorem 11. Let $G=P_{n_{1}} \otimes P_{n_{2}}$ where $n_{1}, n_{2} \geq 2$. Then one has

$$
\eta_{i}(G)= \begin{cases}4, & \text { if } n_{1}=n_{2}=2, \\ m-n+1, & \text { if } n_{1}=2, n_{2}=3, \\ m-n, & \text { otherwise. }\end{cases}
$$

Proof. Let $E(G)=\left\{w_{i j} w_{i(j+1)}: 1 \leq i \leq n_{1} ; 1 \leq j \leq n_{2}-1\right\} \cup$ $\left\{w_{i j} w_{(i+1) j}: 1 \leq i \leq n_{1}-1 ; 1 \leq j \leq n_{2}\right\} \cup\left\{w_{i j} w_{(i+1)(j+1)}:\right.$ $\left.1 \leq i \leq\left(n_{1}-1\right) ; 1 \leq j \leq\left(n_{2}-1\right)\right\} \cup\left\{w_{i j} w_{(i+1)(j-1)}: 1 \leq i \leq\right.$ $\left.\left(n_{1}-1\right) ; 2 \leq j \leq n_{2}\right\}$.

The cases when $n_{1}=n_{2}=2, n_{1}=2$, and $n_{2}=3$ can easily be verified.

Suppose $n_{1}=2$ and $n_{2} \geq 4$. To prove that $\eta_{i}(G)=m-n$, by Remark 4, it is sufficient to obtain an edge-disjoint and internally-disjoint collection $\mathcal{S}$ of induced paths and cycles such that every vertex of $G$ is an internal vertex of an element of $\mathcal{S}$. Let us consider the following cycles and paths of $G$ :

$$
\begin{aligned}
& Q_{1}=\left(w_{22}, w_{21}, w_{11}, w_{22}\right), \\
& Q_{2}=\left(w_{2\left(n_{2}-1\right)}, w_{2 n_{2}}, w_{1 n_{2}}, w_{2\left(n_{2}-1\right)}\right), \\
& Q_{3}=\left(w_{12}, w_{22}, w_{23}, w_{12}\right), \\
& Q_{4}=\left(w_{23}, w_{24}, w_{14}, w_{23}\right), \\
& Q_{5}=\left(w_{1\left(n_{2}-1\right)}, w_{2\left(n_{2}-1\right)}, w_{2\left(n_{2}-2\right)}, w_{1\left(n_{2}-1\right)}\right), \\
& Q_{6}=\left(w_{11}, w_{12}, w_{13}, \ldots, w_{1\left(n_{2}-1\right)}, w_{1 n_{2}}\right), \\
& Q_{7}=\left(w_{11}, w_{12}, w_{13}, w_{14}\right), \\
& Q_{8}=\left(w_{23}, w_{24}, w_{25}, \ldots, w_{2\left(n_{2}-2\right)}\right) .
\end{aligned}
$$


If $n_{2}=4$, let $\mathcal{S}=\left\{Q_{1}, Q_{2}, Q_{3}, Q_{6}\right\}$; if $n_{2}=5$, let $\mathcal{S}=$ $\left\{Q_{1}, Q_{2}, Q_{3}, Q_{4}, Q_{7}\right\}$, and when $n_{2} \geq 6$, let $\mathcal{S}=\left\{Q_{1}, Q_{2}\right.$, $\left.Q_{3}, Q_{5}, Q_{6}, Q_{8}\right\}$ thus obtaining the required collection $\mathcal{S}$, proving that $\eta_{i}(G)=m-n$.

When $n_{1}=n_{2}=3$, we consider the collection $\mathcal{S}=$ $\left\{Q_{1}, Q_{2}, Q_{3}, Q_{4}, Q_{5}\right\}$ where

$$
\begin{aligned}
& Q_{1}=\left(w_{22}, w_{21}, w_{11}, w_{22}\right), \\
& Q_{2}=\left(w_{22}, w_{23}, w_{13}, w_{22}\right), \\
& Q_{3}=\left(w_{21}, w_{31}, w_{32}, w_{21}\right), \\
& Q_{4}=\left(w_{32}, w_{33}, w_{22}, w_{32}\right), \\
& Q_{5}=\left(w_{11}, w_{12}, w_{13}\right) .
\end{aligned}
$$

And for $n_{1} \geq 3$ and $n_{2} \geq 4$, we consider the following paths and cycles

$$
\begin{aligned}
Q_{1} & =\left(w_{22}, w_{11}, w_{12}, w_{22}\right), \\
Q_{2} & =\left(w_{2\left(n_{2}-1\right)}, w_{1 n_{2}}, w_{1\left(n_{2}-1\right)}, w_{2\left(n_{2}-1\right)}\right), \\
Q_{3} & =\left(w_{\left(n_{1}-1\right) 2}, w_{n_{1} 1}, w_{n_{1} 2}, w_{\left(n_{1}-1\right) 2}\right), \\
Q_{4} & =\left(w_{\left(n_{1}-1\right)\left(n_{2}-1\right)}, w_{n_{1} n_{2}}, w_{n_{1}\left(n_{2}-1\right)}, w_{\left(n_{1}-1\right)\left(n_{2}-1\right)}\right), \\
Q_{5} & =\left(w_{11}, w_{21}, w_{31}, \ldots, w_{n_{1} 1}\right) \\
Q_{6} & =\left(w_{1 n_{2}}, w_{2 n_{2}}, w_{3 n_{2}}, \ldots, w_{n_{1} n_{2}}\right), \\
Q_{7} & =\left(w_{12}, w_{13}, w_{14}, \ldots, w_{1\left(n_{2}-1\right)}\right), \\
Q_{8} & =\left(w_{n_{1} 2}, w_{n_{1} 3}, w_{n_{1} 4}, \ldots, w_{n_{1}\left(n_{2}-1\right)}\right), \\
Q_{i-1}^{\prime} & =\left(w_{i 1}, w_{i 2}, w_{i 3}, \ldots, w_{i n_{2}}\right)
\end{aligned}
$$$$
\text { for each } i=2,3,4, \ldots,\left(n_{1}-1\right)
$$

to obtain the collection $\mathcal{S}=\left\{Q_{1}, Q_{2}, \ldots, Q_{8}, Q_{1}^{\prime}, Q_{2}^{\prime}, \ldots\right.$, $\left.Q_{\left(n_{1}-2\right)}^{\prime}\right\}$ mentioned in Remark 4 so that $\eta_{i}(G)=m-n$ thus completing the proof of the theorem.

Theorem 12. For the graph $G=C_{n_{1}} \otimes C_{n_{2}}$ where $n_{1}, n_{2} \geq 3$

$$
\eta_{i}(G)= \begin{cases}m-n+1, & \text { if } n_{1}=n_{2}=3 \\ m-n, & \text { otherwise. }\end{cases}
$$

Proof. We have $E(G)=\left\{w_{i j} w_{i(j+1)}: 1 \leq i \leq n_{1} ; 1 \leq j \leq\right.$ $\left.n_{2}-1\right\} \cup\left\{w_{i n_{2}} w_{i 1}: 1 \leq i \leq n_{1}\right\} \cup\left\{w_{i n_{2}} w_{(i+1) 1}: 1 \leq i \leq\left(n_{1}-1\right)\right\} \cup$ $\left\{w_{i 1} w_{(i+1) n_{2}}: 1 \leq i \leq\left(n_{1}-1\right)\right\} \cup\left\{w_{i j} w_{(i+1) j}: 1 \leq i \leq n_{1}-1 ; 1 \leq\right.$ $\left.j \leq n_{2}\right\} \cup\left\{w_{n_{1} j} w_{1 j}: 1 \leq j \leq n_{2}\right\} \cup\left\{w_{1 j} w_{n_{1}(j+1)}: 1 \leq i \leq\right.$ $\left.\left(n_{2}-1\right)\right\} \cup\left\{w_{n_{1} j} w_{1(j+1)}: 1 \leq i \leq\left(n_{2}-1\right)\right\} \cup\left\{w_{i j} w_{(i+1)(j+1)}:\right.$ $\left.1 \leq i \leq\left(n_{1}-1\right) ; 1 \leq j \leq\left(n_{2}-1\right)\right\} \cup\left\{w_{i j} w_{(i+1)(j-1)}: 1 \leq i \leq\right.$ $\left.\left(n_{1}-1\right) ; 2 \leq j \leq n_{2}\right\} \cup\left\{w_{11} w_{n_{1} n_{2}}, w_{1 n_{2}} w_{n_{1} 1}\right\}$.
When $n_{1}=n_{2}=3$ clearly $G=K_{9}$, and therefore by Theorem $5, \eta_{i}(G)=m-n+1$. If $n_{1} \geq 3$ and $n_{2} \geq 4$, consider

$$
\begin{aligned}
Q_{1} & =\left(w_{11}, w_{12}, w_{13}, \ldots, w_{1 n_{2}}, w_{11}\right), \\
Q_{2} & =\left(w_{1 n_{2}}, w_{2 n_{2}}, w_{3 n_{2}}, \ldots, w_{n_{1} n_{2}}, w_{1 n_{2}}\right), \\
Q_{3} & =\left(w_{n_{1} n_{2}}, w_{n_{1}\left(n_{2}-1\right)}, w_{n_{1}\left(n_{2}-2\right)}, \ldots, w_{n_{1} 1}, w_{n_{1} n_{2}}\right), \\
Q_{4} & =\left(w_{n_{1} 1}, w_{\left(n_{1}-1\right) 1}, w_{\left(n_{1}-2\right) 1}, \ldots, w_{11}, w_{n_{1} 1}\right), \\
Q_{5} & =\left(w_{11}, w_{22}, w_{23}, w_{11}\right), \\
Q_{6} & =\left(w_{22}, w_{32}, w_{42}, \ldots, w_{n_{1} 2}\right), \\
Q_{7} & =\left(w_{23}, w_{33}, w_{43}, \ldots, w_{n_{1} 3}\right), \\
Q_{i-1}^{\prime} & =\left(w_{i 3}, w_{i 4}, w_{i 5}, \ldots, w_{i\left(n_{2}-1\right)}, w_{i n_{2}}\right) ; \quad 2 \leq i \leq\left(n_{1}-1\right) .
\end{aligned}
$$

Then $\mathcal{S}=\left\{Q_{1}, Q_{2}, \ldots, Q_{7}, Q_{1}^{\prime}, Q_{2}^{\prime}, \ldots, Q_{\left(n_{1}-2\right)}^{\prime}\right\}$ forms an internally-disjoint and edge-disjoint collection of induced paths and cycles having all the vertices of $G$ as internal vertices. Hence by Remark 4 we have the desired result.

Theorem 13. If $G=P_{n_{1}} \otimes C_{n_{2}}$, where $n_{1} \geq 2$ and $n_{2} \geq 3$, then $\eta_{i}(G)=m-n$.

Proof. The edge set of $G$ is given by $E(G)=\left\{w_{i j} w_{i(j+1)}: 1 \leq\right.$ $\left.i \leq n_{1} ; 1 \leq j \leq n_{2}-1\right\} \cup\left\{w_{i n_{2}} w_{i 1}: 1 \leq i \leq n_{1}\right\} \cup\left\{w_{i n_{2}} w_{(i+1) 1}:\right.$ $\left.1 \leq i \leq\left(n_{1}-1\right)\right\} \cup\left\{w_{i 1} w_{(i+1) n_{2}}: 1 \leq i \leq\left(n_{1}-1\right)\right\} \cup\left\{w_{i j} w_{(i+1) j}:\right.$ $\left.1 \leq i \leq n_{1}-1 ; 1 \leq j \leq n_{2}\right\} \cup\left\{w_{i j} w_{(i+1)(j+1)}: 1 \leq i \leq\left(n_{1}-\right.\right.$ $\left.1) ; 1 \leq j \leq\left(n_{2}-1\right)\right\} \cup\left\{w_{i j} w_{(i+1)(j-1)}: 1 \leq i \leq\left(n_{1}-1\right) ; 2 \leq\right.$ $\left.j \leq n_{2}\right\}$.

In this case also, we will show the existence of a collection $\delta$ of internally-disjoint and edge-disjoint induced paths and cycles in $G$ such that each vertex of $G$ is an internal vertex of an element of $\mathcal{S}$ so that by Remark 4 , we get $\eta_{i}(G)=m-n$.

When $n_{1}=2$, consider

$$
\begin{aligned}
& Q^{\prime}=\left(w_{1 n_{2}}, w_{2 n_{2}}, w_{11}, w_{1 n_{2}}\right), \\
& Q_{j}=\left(w_{1 j}, w_{2 j}, w_{1(j+1)}, w_{1 j}\right) \\
& \quad \quad \text { for each } j=1,2, \ldots,\left(n_{2}-1\right)
\end{aligned}
$$

to obtain the desired collection $\mathcal{S}=\left\{Q^{\prime}, Q_{1}, Q_{2}, \ldots, Q_{\left(n_{2}-1\right)}\right\}$ of induced cycles. Next we consider

$$
\begin{aligned}
& Q_{1}=\left(w_{21}, w_{11}, w_{1 n_{2}}, w_{21}\right), \\
& Q_{2}=\left(w_{21}, w_{31}, w_{3 n_{2}}, w_{21}\right), \\
& Q_{3}=\left(w_{12}, w_{21}, w_{32}\right), \\
& Q_{4}=\left(w_{11}, w_{12}, w_{22}, w_{11}\right),
\end{aligned}
$$




$$
\begin{aligned}
Q_{5} & =\left(w_{22}, w_{23}, w_{32}, w_{22}\right), \\
Q_{1}^{\prime} & =\left(w_{12}, w_{13}, \ldots, w_{1 n_{2}}\right), \\
Q_{2}^{\prime} & =\left(w_{32}, w_{33}, \ldots, w_{3 n_{2}}\right), \\
Q_{(j-1)}^{\prime} & =\left(w_{1 j}, w_{2 j}, w_{3 j}\right), \text { for each } j=4,5, \ldots, n_{2}
\end{aligned}
$$

so that when $n_{1}=n_{2}=3, \mathcal{S}=\left\{Q_{1}, Q_{2}, Q_{3}, Q_{4}, Q_{5}\right\}$ and when $n_{1}=3, n_{2} \geq 4, \mathcal{S}=\left\{Q_{1}, Q_{2}, \ldots, Q_{5}, Q_{1}^{\prime}, Q_{2}^{\prime}, \ldots, Q_{\left(n_{2}-1\right)}^{\prime}\right\}$ form the required collection of edge-disjoint and internallydisjoint induced paths/cycles.

If $n_{1} \geq 4$, we consider

$$
\begin{aligned}
& Q_{1}=\left(w_{21}, w_{11}, w_{1 n_{2}}, w_{21}\right), \\
& Q_{2}=\left(w_{\left(n_{1}-1\right) 1}, w_{n_{1} 1}, w_{n_{1} n_{2}}, w_{\left(n_{1}-1\right) 1}\right), \\
& Q_{3}=\left(w_{12}, w_{21}, w_{31}, \ldots, w_{\left(n_{1}-1\right) 1}, w_{n_{1} 2}\right), \\
& Q_{4}=\left(w_{11}, w_{12}, w_{22}, w_{11}\right), \\
& Q_{5}=\left(w_{n_{1} 1}, w_{n_{1} 2}, w_{\left(n_{1}-1\right) 2}, w_{n_{1} 1}\right), \\
& Q_{1}^{\prime}=\left(w_{12}, w_{13}, \ldots, w_{1 n_{2}}\right), \\
& Q_{2}^{\prime}=\left(w_{n_{1} 2}, w_{n_{1} 3}, \ldots, w_{n_{1} n_{2}}\right), \\
& Q_{j}^{\prime}=\left(w_{1 j}, w_{2 j}, \ldots, w_{n_{1} j}\right), \quad \text { for each } j=3,4, \ldots n_{2}
\end{aligned}
$$

to obtain the collection $\mathcal{S}=\left\{Q_{1}, Q_{2}, \ldots, Q_{5}, Q_{1}^{\prime}, Q_{2}^{\prime}, \ldots, Q_{n_{2}}^{\prime}\right\}$ so that by Remark $4 \eta_{i}(G)=m-n$, thus completing the proof.

Theorem 14. Let $G=P_{n_{1}} \circ P_{n_{2}}$, where $n_{1}, n_{2} \geq 2$ such that $n_{2} \geq n_{1}$. Then

$$
\eta_{i}(G)= \begin{cases}4, & \text { if } n_{1}=n_{2}=2, \\ m-n, & \text { otherwise. }\end{cases}
$$

Proof. We know that $E(G)=\left\{w_{i j} w_{(i+1) k}: 1 \leq i \leq n_{1}-1 ; 1 \leq\right.$ $\left.j \leq n_{2} ; 1 \leq k \leq n_{2}\right\} \cup\left\{w_{i j} w_{i(j+1)}: 1 \leq i \leq n_{1} ; 1 \leq j \leq\right.$ $\left.\left(n_{2}-1\right)\right\}$.

When $n_{1}=n_{2}=2, G=K_{4}$, and therefore by Theorem 5 , $\eta_{i}(G)=4$. If $n_{1}=2$ and $n_{2} \geq 3$, consider

$$
\begin{aligned}
& Q_{j}=\left(w_{1 j}, w_{2 j}, w_{1(j+1)}, w_{1 j}\right) \quad \text { where } 1 \leq j \leq\left(n_{2}-1\right), \\
& Q^{\prime}=\left(w_{2\left(n_{2}-1\right)}, w_{2 n_{2}}, w_{11}, w_{2\left(n_{2}-1\right)}\right) .
\end{aligned}
$$

Then the collection $\mathcal{S}=\left\{Q_{1}, Q_{2}, \ldots, Q_{\left(n_{2}-1\right)}, Q^{\prime}\right\}$ of internally-disjoint induced paths and cycles satisfies the property mentioned in Remark 4 and therefore $\eta_{i}(G)=m-n$.

When $n_{1}, n_{2} \geq 3$, we observe that all the edges of the strong product $P_{n_{1}} \otimes P_{n_{2}}$ are included in $E\left(P_{n_{1}} \circ P_{n_{2}}\right)$. Therefore using the same argument as that of Theorem 11 we get $\eta_{i}(G)=$ $m-n$ thus completing the proof of the theorem.
Theorem 15. For the graph $G=C_{n_{1}} \circ C_{n_{2}}$, where $n_{1}, n_{2} \geq 3$ with $n_{2} \geq n_{1}$ one has

$$
\eta_{i}(G)= \begin{cases}m-n+1, & \text { if } n_{1}=n_{2}=3 \\ m-n, & \text { otherwise. }\end{cases}
$$

Proof. For the given graph $G$ the edge set $E(G)=\left\{w_{i j} w_{(i+1) k}\right.$ : $\left.1 \leq i \leq\left(n_{1}-1\right) ; 1 \leq j \leq n_{2} ; 1 \leq k \leq n_{2}\right\} \cup\left\{w_{i j} w_{i(j+1)}: 1 \leq\right.$ $\left.i \leq n_{1} ; 1 \leq j \leq\left(n_{2}-1\right)\right\} \cup\left\{w_{i 1} w_{i n_{2}}: 1 \leq i \leq n_{1}\right\} \cup\left\{w_{1 j} w_{n_{1} k}\right.$ : $\left.1 \leq j \leq n_{2} ; 1 \leq k \leq n_{2}\right\}$.

When $n_{1}=n_{2}=3, G=K_{9}$, and therefore by Theorem 5, $\eta_{i}(G)=m-n+1$. If $n_{1} \geq 3$ and $n_{2} \geq 4$, then the same set $\mathcal{S}=\left\{Q_{1}, Q_{2}, \ldots, Q_{7}, Q_{1}^{\prime}, Q_{2}^{\prime}, \ldots, Q_{\left(n_{1}-2\right)}^{\prime}\right\}$ we have considered in Theorem 12 is sufficient to prove that $\eta_{i}(G)=m-n$.

Remark 16. The lexicographic product of two graphs is not commutative. Therefore the case when $n_{1}>n_{2}$ is to be considered separately. However, using the same argument followed in the previous theorems, it is not difficult to show that $\eta_{i}\left(P_{n_{1}} \circ P_{n_{2}}\right)=m-n+1$ when $n_{1}=3$ and $n_{2}=2$ whereas in all other cases $\eta_{i}(G)=m-n$, whether $G=P_{n_{1}} \circ P_{n_{2}}$ or $G=C_{n_{1}} \circ C_{n_{2}}$

Theorem 17. Let $G=P_{n_{1}} \circ C_{n_{2}}$, where $n_{1} \geq 2$ and $n_{2} \geq 3$. Then $\eta_{i}(G)=m-n$.

Proof. The edge set of $G$ is given by $E(G)=\left\{w_{i j} w_{(i+1) k}: 1 \leq\right.$ $\left.i \leq\left(n_{1}-1\right) ; 1 \leq j \leq n_{2} ; 1 \leq k \leq n_{2}\right\} \cup\left\{w_{i j} w_{i(j+1)}: 1 \leq i \leq\right.$ $\left.n_{1} ; 1 \leq j \leq\left(n_{2}-1\right)\right\} \cup\left\{w_{i 1} w_{i n_{2}}: 1 \leq i \leq n_{1}\right\}$.

This result follows immediately from Theorem 13 as the collection of induced paths and cycles considered in the set $\mathcal{S}$ in various cases there is applicable to the graph $G=P_{n_{1}} \circ C_{n_{2}}$ as well.

Remark 18. It can also be proved that $\eta_{i}\left(C_{n_{2}} \circ P_{n_{1}}\right)=m-$ $n$ by showing the existence of a collection $\mathcal{S}$ mentioned in Remark 4.

\section{Induced Acyclic Graphoidal Decomposition Number of Products of Paths and Cycles}

Many of the path decomposition problems in the literature deal with splitting of a graph into paths (and not cycles) with a specified property. Therefore in this section we consider the concept of induced acyclic graphoidal decomposition for product graphs. As seen before, an induced acyclic graphoidal decomposition (IAGD) of a graph $G$ is an induced graphoidal decomposition $\psi$ where each member of $\psi$ is an induced path. The minimum cardinality of an IAGD of $G$ is called the induced acyclic graphoidal decomposition number of $G$, and it is denoted by $\eta_{i a}(G)$.

As in the previous section, we determine the value of $\eta_{i a}$ for nine types of product graphs. We begin with the Cartesian product of two paths. 
Theorem 19. For the graph $G=P_{n_{1}} \square P_{n_{2}}$ where $n_{1}, n_{2} \geq 2$,

$$
\eta_{i a}(G)= \begin{cases}m-n+2, & \text { if } n_{1}=2, n_{2} \geq 2 \text { or } n_{1}=n_{2}=3 \\ m-n+1, & \text { if } n_{1}=3 \text { or } 4, n_{2}=4 \\ m-n, & \text { otherwise. }\end{cases}
$$

Proof. If $n_{1}=n_{2}=2, G=C_{4}$, and so, $\eta_{i a}(G)=2=$ $m-n+2$. For $n_{1}=2$ and $n_{2} \geq 3$, let $Q_{1}=\left(w_{11}\right.$, $\left.w_{21}, w_{22}, w_{23}, \ldots, w_{2 n_{2}}, w_{1 n_{2}}\right)$, let $Q_{2}=\left(w_{11}, w_{12}, w_{13}, \ldots\right.$, $\left.w_{1 n_{2}}\right)$, and let $S^{\prime}=\left\{w_{1 j} w_{2 j}: 2 \leq j \leq\left(n_{2}-1\right)\right\}$. Then $Q_{1}$ and $Q_{2}$ along with the set $S^{\prime}$ form an IAGD $\psi$ of $G$ with cardinality $n_{2}=m-n+2$ so that $\eta_{i a}(G) \leq|\psi|=m-n+2$. Also for any IAGD $\psi$ of $G$, one of the vertices $w_{11}$ and $w_{21}$ will be exterior and the same is in the case with the vertices $w_{1 n_{2}}$ and $w_{2 n_{2}}$. Therefore, $t_{i a}(G)$ - the maximum number of vertices interior to any IAGD of $G-$ is $\leq(n-2)$, and hence by Theorem $6 \eta_{i a}(G) \geq m-n+2$ proving the desired result.

The case when $n_{1}=n_{2}=3$ also is similar to the previous case where at least two vertices of $G$ will be exterior to any IAGD of $G$, and, therefore, $\eta_{i a}(G) \geq m-n+2$ by Theorem 6 . Further, the paths

$$
\begin{aligned}
& Q_{1}=\left(w_{11}, w_{21}, w_{31}, w_{32}, w_{33}, w_{23}, w_{13}\right), \\
& Q_{2}=\left(w_{11}, w_{12}, w_{13}\right) \\
& Q_{3}=\left(w_{21}, w_{22}, w_{23}\right)
\end{aligned}
$$

along with the edges $w_{12} w_{22}$ and $w_{22} w_{32}$ form an IAGD of cardinality $5=m-n+2$, and hence it follows that $\eta_{i a}(G)=$ $m-n+2$.

Next assume that $n_{1}=3$ and $n_{2}=4$. Consider the induced paths $Q_{1}, Q_{2}$, and $Q_{3}$, where

$$
\begin{aligned}
& Q_{1}=\left(w_{11}, w_{21}, w_{31}, w_{32}, w_{33}, w_{34}, w_{24}, w_{14}, w_{13}\right), \\
& Q_{2}=\left(w_{11}, w_{12}, w_{13}, w_{23}\right), \\
& Q_{3}=\left(w_{21}, w_{22}, w_{23}, w_{24}\right) .
\end{aligned}
$$

Then the collection $\psi_{1}=\left\{Q_{1}, Q_{2}, Q_{3}\right\}$ together with the edges $e_{1}=w_{12} w_{22}, e_{2}=w_{22} w_{32}$, and $e_{3}=w_{23} w_{33}$ forms an IAGD $\psi$ of $G$ such that $|\psi|=6=m-n+1$ proving that $\eta_{i a} \leq m-n+1$. Also it can be verified that at least one vertex of $G$ is exterior to any IAGD of $G$, and therefore $\eta_{i a} \geq m-n+1$ by Theorem 6 thus proving the required result.

If $n_{1}=4=n_{2}$, then as in the previous case any IAGD of $G$ will have at least one vertex exterior to it so that $\eta_{i a}(G) \geq m-$ $n+1$. Also there exists an IAGD $\psi$ of cardinality $9=m-n+1$ consisting of the induced paths $Q_{1}, Q_{2}, Q_{3}$, and $Q_{4}$ where

$$
\begin{aligned}
& Q_{1}=\left(w_{11}, w_{21}, w_{31}, w_{41}, w_{42}, w_{43}, w_{44}, w_{34}, w_{24}, w_{14}, w_{13}\right), \\
& Q_{2}=\left(w_{11}, w_{12}, w_{13}, w_{23}\right), \\
& Q_{3}=\left(w_{21}, w_{22}, w_{23}, w_{24}\right), \\
& Q_{4}=\left(w_{31}, w_{32}, w_{33}, w_{34}\right)
\end{aligned}
$$

and the edges $e_{1}=w_{12} w_{22}, e_{2}=w_{22} w_{32}, e_{3}=w_{32} w_{42}, e_{4}=$ $w_{23} w_{33}$, and $e_{5}=w_{33} w_{43}$ so that $\eta_{i a} \leq m-n+1$ and hence the result.

Finally, assume that $n_{1} \geq 3$ and $n_{2} \geq 5$. Consider the paths

$$
\begin{gathered}
Q_{1}=\left(w_{12}, w_{11}, w_{21}, \ldots, w_{n_{1} 1}, w_{n_{1} 2}, \ldots,\right. \\
\left.w_{n_{1} n_{2}}, w_{\left(n_{1}-1\right) n_{2}}, w_{1 n_{2}}, w_{1\left(n_{2}-1\right)}\right), \\
Q^{\prime}=\left(w_{22}, w_{12}, w_{13}, \ldots, w_{1\left(n_{2}-1\right)}, w_{2\left(n_{2}-1\right)}\right), \\
Q_{i}=\left(w_{i 1}, w_{i 2}, \ldots, w_{i n_{2}}\right)
\end{gathered}
$$$$
\text { for each } i=2,3, \ldots\left(n_{1}-1\right) \text {. }
$$

Then $\mathcal{S}=\left\{Q_{1}, Q_{2}, \ldots, Q_{\left(n_{1}-1\right)}, Q^{\prime}\right\}$ is an edge-disjoint collection of internally-disjoint induced paths of $G$ such that every vertex of $G$ is an internal vertex of some path in $\mathcal{S}$. Therefore by $\operatorname{Remark} 7, \eta_{i a}(G)=m-n$.

Theorem 20. If $G=C_{n_{1}} \square C_{n_{2}}$, where $n_{1}, n_{2} \geq 3$, then $\eta_{i a}(G)=m-n$.

Proof. For the given graph we will show that there exists a collection $\mathcal{S}$ of internally-disjoint and edge-disjoint induced paths such that each vertex of $G$ is internal to a member of $\mathcal{S}$. If $n_{1}=3$, let $\mathcal{S}=\left\{Q_{1}, Q_{2}, Q_{3}, Q_{4}\right\}$ where

$$
\begin{gathered}
Q_{1}=\left(w_{21}, w_{11}, w_{1 n_{2}}, w_{3 n_{2}}, w_{3\left(n_{2}-1\right)}, \ldots, w_{32}\right), \\
Q_{2}=\left(w_{11}, w_{12}, \ldots, w_{1\left(n_{2}-1\right)}, w_{2\left(n_{2}-1\right)}, w_{2 n_{2}}, w_{3 n_{2}}\right), \\
Q_{3}=\left(w_{2\left(n_{2}-1\right)}, w_{2\left(n_{2}-2\right)}, \ldots, w_{21}, w_{31}, w_{3 n_{2}}\right), \\
Q_{4}=\left(w_{31}, w_{32}, w_{23}\right) . \\
\text { If } n_{1} \geq 4, \text { let } \\
Q_{1}=\left(w_{21}, w_{11}, w_{1 n_{2}}, w_{n_{1} n_{2}}, w_{n_{1}\left(n_{2}-1\right)}, \ldots, w_{n_{1} 2}\right), \\
Q_{2}=\left(w_{11}, w_{12}, \ldots, w_{1\left(n_{2}-1\right)}, w_{2\left(n_{2}-1\right)}, w_{2 n_{2}}, w_{3 n_{2}}, \ldots, w_{n_{1} n_{2}}\right), \\
Q_{3}=\left(w_{2\left(n_{2}-1\right)}, w_{2\left(n_{2}-2\right)}, \ldots, w_{21}, w_{31}, \ldots, w_{n_{1} 1}, w_{n_{1} n_{2}}\right), \\
Q_{4}=\left(w_{n_{1} 1}, w_{n_{1} 2}, w_{\left(n_{1}-1\right) 2}, w_{\left(n_{1}-1\right) 3}, \ldots, w_{\left(n_{1}-1\right) n_{2}}\right), \\
Q_{i-1}^{\prime}=\left(w_{2 j}, w_{3 j}, \ldots, w_{\left(n_{1}-1\right) j}\right) \quad \text { for each } j=2,3, \ldots,\left(n_{2}-1\right) .
\end{gathered}
$$

Then $\mathcal{S}=\left\{Q_{1}, Q_{2}, Q_{3}, Q_{4}, Q_{1}^{\prime}, Q_{2}^{\prime}, \ldots, Q_{\left(n_{2}-2\right)}^{\prime}\right\}$ forms the desired collection of internally-disjoint and edge-disjoint induced paths such that all the vertices of $G$ are internal to some path in $\mathcal{S}$, and by $\operatorname{Remark} 7, \eta_{i a}(G)=m-n$.

Theorem 21. If $G=P_{n_{1}} \square C_{n_{2}}$, then $\eta_{i a}(G)=m-n$.

Proof. The result follows from Theorem 10 and Remark 7 as the collection $\mathcal{S}$ considered there does not contain any cycle. 
Theorem 22. Let $G=P_{n_{1}} \otimes P_{n_{2}}$ where $n_{1}, n_{2} \geq 2$. Then one has

$$
\eta_{i a}(G)= \begin{cases}6, & \text { if } n_{1}=n_{2}=2 \\ m-n+4, & \text { otherwise. }\end{cases}
$$

Proof. When $n_{1}=n_{2}=2$, the result is immediate as $G=K_{4}$ which has its edge set as a minimum IAGD as $G$ does not contain any induced paths of length greater than one.

In all other cases it can easily be observed that the vertices $w_{11}, w_{1 n_{1}}, w_{n_{1} 1}$, and $w_{n_{1} n_{2}}$ cannot be made interior to any IAGD of $G$. Therefore $t_{i a}(G) \leq n-4$, and hence by Theorem 6 , $\eta_{i a} \geq m-n+4$. Now, let

$$
\begin{aligned}
Q_{i} & =\left(w_{i 1}, w_{i 2}, \ldots, w_{i n_{2}}\right) \text { for each } i=1,2, \ldots, n_{1}, \\
Q^{\prime} & =\left(w_{11}, w_{21}, \ldots, w_{n_{1} 1}\right), \\
Q^{\prime \prime} & =\left(w_{1 n_{2}}, w_{2 n_{2}}, \ldots, w_{n_{1} n_{2}}\right) .
\end{aligned}
$$

Then the collection of induced paths $S_{1}=$ $\left\{Q_{1}, Q_{2}, \ldots, Q_{n_{1}}, Q^{\prime}, Q^{\prime \prime}\right\}$ together with the edges of $G$ not covered by the paths in $S_{1}$ forms an IAGD $\psi$ such that $|\psi|=n_{1}+2\left(n_{2}-2\right)\left(n_{1}-1\right)+2\left(n_{1}-1\right)\left(n_{2}-1\right)=m-n+4$, proving that $\eta_{i a}(G) \leq m-n+4$, and this completes the proof of the theorem.

Theorem 23. If $G$ is the graph $C_{n_{1}} \otimes C_{n_{2}}$ where $n_{1}, n_{2} \geq 3$, then

$$
\eta_{i a}(G)= \begin{cases}m, & \text { if } n_{1}=n_{2}=3 \\ m-n, & \text { otherwise. }\end{cases}
$$

Proof. When $n_{1}=n_{2}=3$, we have $G=K_{9}$, and therefore $\eta_{i}(G)=m$ as the edge set of $G$ is a minimum IAGD of $G$.

If $n_{1}=3$ and $n_{2}=4$, we consider the collection of induced paths $\mathcal{S}=\left\{\left(w_{12}, w_{11}, w_{14}\right),\left(w_{22}, w_{21}, w_{24}\right),\left(w_{32}, w_{31}, w_{34}\right)\right.$, $\left(w_{21}, w_{12}, w_{23}\right), \quad\left(w_{31}, w_{22}, w_{33}\right), \quad\left(w_{21}, w_{32}, w_{23}\right), \quad\left(w_{12}, w_{13}\right.$, $\left.w_{24}\right),\left(w_{22}, w_{23}, w_{34}\right),\left(w_{32}, w_{33}, w_{24}\right),\left(w_{13}, w_{14}, w_{31}\right),\left(w_{23}\right.$, $\left.\left.w_{24}, w_{31}\right),\left(w_{33}, w_{34}, w_{11}\right)\right\}$ which satisfy the property mentioned in Remark 7, and therefore $\eta_{i a}(G)=m-n$ in this case.

If $n_{1} \geq 3$ and $n_{2} \geq 5$, we consider the following induced paths:

$$
\begin{aligned}
Q_{i} & =\left(w_{i 2}, w_{i 1}, w_{i n_{2}}, w_{i\left(n_{2}-1\right)}\right) \text { for each } i=1,2, \ldots, n_{1}, \\
Q_{i}^{\prime} & =\left(w_{(i+1) 1}, w_{i 2}, w_{(i+1) 3}\right) \text { for each } i=1,2, \ldots,\left(n_{1}-1\right), \\
Q^{*} & =\left(w_{\left(n_{1}-1\right) 1}, w_{n_{1} 2}, w_{\left(n_{1}-1\right) 3}\right) \\
Q_{i}^{\prime \prime} & =\left(w_{(i+1)\left(n_{2}-2\right)}, w_{i\left(n_{2}-1\right)}, w_{(i+1) n_{2}}\right) \\
Q^{* *} & =\left(w_{\left(n_{1}-1\right)\left(n_{2}-2\right)}, w_{n_{1}\left(n_{2}-1\right)}, w_{\left(n_{1}-1\right) n_{2}}\right) \\
Q_{i}^{\prime \prime \prime} & =\left(w_{i 2}, w_{i 3}, \ldots, w_{i\left(n_{2}-1\right)}\right) \text { for each } i=1,2, \ldots,\left(n_{1}-1\right)
\end{aligned}
$$

Then the collection $\mathcal{S}$ of all the paths given previous constitutes an edge-disjoint collection of internally-disjoint induced paths in $G$ such that each vertex of $G$ is internal to some path in $\mathcal{S}$. Therefore it follows from Remark 7 that $\eta_{i a}=m-n$, thus completing the proof.

Theorem 24. Let $G=P_{n_{1}} \otimes C_{n_{2}}$ where $n_{1} \geq 2 ; n_{2} \geq 3$. Then

$$
\eta_{i a}(G)= \begin{cases}m-n+6, & \text { if } n_{2}=3, \\ m-n, & \text { otherwise. }\end{cases}
$$

Proof. Suppose $n_{2}=3$. Then it can be observed that the vertices $w_{11}, w_{12}, w_{13}, w_{n_{1} 1}, w_{n_{1} 2}$ and $w_{n_{1} 3}$ cannot be made internal to any induced path of $G$. Therefore, $t_{i a}(G) \leq(n-6)$. Further, the three paths $Q_{1}=\left\{w_{11}, w_{21}, \ldots, w_{n_{1} 1}\right\} ; Q_{2}=$ $\left\{w_{12}, w_{22}, \ldots, w_{n_{1} 2}\right\}$, and $Q_{3}=\left\{w_{13}, w_{23}, \ldots, w_{n_{1}}\right\}$ together with the edges of $G$ not belonging to the induced paths $Q_{1}$, $Q_{2}$, and $Q_{3}$ form an IAGD of cardinality $m-3\left(n_{1}-1\right)+3=$ $m-n+6$ so that $\eta_{i a}(G) \leq m-n+6$ thus proving that $\eta_{i a}(G)=m-n+6$.

Next assume that $n_{2} \geq 4$. If $n_{1}=2$, consider the induced paths

$$
\begin{aligned}
& Q_{1}=\left(w_{12}, w_{11}, w_{1 n_{2}}\right), \\
& Q_{2}=\left(w_{21}, w_{12}, w_{23}\right), \\
& Q_{3}=\left(w_{22}, w_{21}, w_{2 n_{2}}\right), \\
& Q_{4}=\left(w_{11}, w_{22}, w_{13}\right), \\
& Q_{5}=\left(w_{21}, w_{1 n_{2}}, w_{2\left(n_{2}-1\right)}\right), \\
& Q_{6}=\left(w_{11}, w_{2 n_{2}}, w_{1\left(n_{2}-1\right)}\right), \\
& Q_{7}=\left(w_{12}, w_{13}, \ldots, w_{1 n_{2}}\right), \\
& Q_{8}=\left(w_{22}, w_{23}, \ldots, w_{2 n_{2}}\right)
\end{aligned}
$$

so that $\mathcal{S}=\left\{Q_{1}, Q_{2}, \ldots, Q_{8}\right\}$ is a collection of edge-disjoint and internally-disjoint induced paths such that each vertex of $G$ is an internal vertex of a path in $\delta$. Therefore by Remark 7 , $\eta_{i a}(G)=m-n$.

If $n_{1} \geq 3$ and $n_{2} \geq 4$, let

$$
\begin{aligned}
& Q_{1}=\left(w_{12}, w_{11}, w_{1 n_{2}}\right), \\
& Q_{2}=\left(w_{21}, w_{12}, w_{23}\right), \\
& Q_{3}=\left(w_{21}, w_{1 n_{2}}, w_{2\left(n_{2}-1\right)}\right), \\
& Q_{4}=\left(w_{n_{1} 2}, w_{n_{1} 1}, w_{n_{1} n_{2}}\right), \\
& Q_{5}=\left(w_{\left(n_{1}-1\right) 1}, w_{n_{1} 2}, w_{\left(n_{1}-1\right) 3}\right), \\
& Q_{6}=\left(w_{\left(n_{1}-1\right) 1}, w_{n_{1} n_{2}}, w_{\left(n_{1}-1\right)\left(n_{2}-1\right)}\right), \\
& Q_{7}=\left(w_{12}, w_{13}, \ldots, w_{1 n_{2}}\right),
\end{aligned}
$$




$$
\begin{aligned}
Q_{8} & =\left(w_{n_{1} 2}, w_{n_{1} 3}, \ldots, w_{n_{1} n_{2}}\right) \\
Q_{(j-1)}^{\prime} & =\left(w_{1 j}, w_{2 j}, \ldots, w_{n_{1} j}\right) \text { for each } j=2,3, \ldots, n_{2} .
\end{aligned}
$$

Then $\mathcal{S}=\left\{Q_{1}, Q_{2}, \ldots, Q_{8}, Q_{1}^{\prime}, Q_{2}^{\prime}, \ldots, Q_{\left(n_{2}-1\right)}^{\prime}\right\}$ is a collection of internally-disjoint and edge-disjoint induced paths mentioned in Remark 7 so that $\eta_{i a}(G)=m-n$.

Theorem 25. For the graph $G=P_{n_{1}} \circ P_{n_{2}}$ where $n_{1}, n_{2} \geq 2$ and $n_{2} \geq n_{1}$ one has

$$
\eta_{i a}(G)= \begin{cases}m, & \text { if } n_{1}=n_{2}=2, \\ m-n+2, & \text { if } n_{1}=2, n_{2}=3, \\ m-n, & \text { otherwise. }\end{cases}
$$

Proof. When $n_{1}=n_{2}=2, G=K_{4}$, and therefore $\eta_{i a}(G)=m$.

Next assume that $n_{1}=2$ and $n_{2}=3$. Then at least two of the vertices $w_{11}, w_{1 n_{2}}, w_{21}, w_{2 n_{2}}$ will be exterior to any IAGD of $G$, and hence $\eta_{i a} \geq m-n+2$ by Theorem 6 . Now, let

$$
\begin{aligned}
& Q_{1}=\left(w_{11}, w_{21}, w_{13}\right), \\
& Q_{2}=\left(w_{11}, w_{22}, w_{13}\right), \\
& Q_{3}=\left(w_{11}, w_{23}, w_{13}\right), \\
& Q_{4}=\left(w_{21}, w_{12}, w_{23}\right) .
\end{aligned}
$$

Then the collection $\psi^{\prime}=\left\{Q_{1}, Q_{2}, Q_{3}, Q_{4}\right\}$ along with the edges of $G$ not covered by the paths in $\psi^{\prime}$ forms an IAGD of cardinality $m-n+2$, and hence it follows that $\eta_{i a}=m-n+2$.

If $n_{1}=2$ and $n_{2} \geq 4$, then we get a collection $\mathcal{S}$ of induced paths given by $\mathcal{S}=\left\{Q_{1}, Q_{2}, \ldots, Q_{6}\right\}$ which satisfies the condition mentioned in Remark 7 by considering the following paths:

$$
\begin{aligned}
& Q_{1}=\left(w_{21}, w_{11}, w_{23}\right), \\
& Q_{2}=\left(w_{22}, w_{1 n_{2}}, w_{2 n_{2}}\right), \\
& Q_{3}=\left(w_{12}, w_{21}, w_{14}\right), \\
& Q_{4}=\left(w_{11}, w_{2 n_{2}}, w_{13}\right), \\
& Q_{5}=\left(w_{11}, w_{12}, \ldots, w_{1 n_{2}}\right), \\
& Q_{6}=\left(w_{21}, w_{22}, \ldots, w_{2 n_{2}}\right) .
\end{aligned}
$$

Thus we have $\eta_{i a}(G)=m-n$. Next assume that $n_{1}=3$ and $n_{2} \geq 3$. In this case let us consider the paths

$$
\begin{aligned}
& Q_{1}=\left(w_{21}, w_{11}, w_{2 n_{2}}\right), \\
& Q_{2}=\left(w_{21}, w_{1 n_{2}}, w_{2 n_{2}}\right), \\
& Q_{3}=\left(w_{21}, w_{31}, w_{2 n_{2}}\right),
\end{aligned}
$$

$$
\begin{aligned}
& Q_{4}=\left(w_{21}, w_{3 n_{2}}, w_{2 n_{2}}\right), \\
& Q_{5}=\left(w_{12}, w_{21}, w_{32}\right), \\
& Q_{6}=\left(w_{12}, w_{2 n_{2}}, w_{32}\right), \\
& Q_{i}^{\prime}=\left(w_{i 1}, w_{i 2}, \ldots, w_{i n_{2}}\right) \text { for each } i=1,2,3 .
\end{aligned}
$$

Then $\mathcal{S}=\left\{Q_{1}, Q_{2}, \ldots, Q_{6}, Q_{1}^{\prime}, Q_{2}^{\prime}, Q_{3}^{\prime}\right\}$ is an internallydisjoint and edge-disjoint collection of induced paths in $G$ such that each vertex of $G$ is internal to some path in $\mathcal{S}$ and so by Remark 7, $\eta_{i a}=m-n$. Finally, when $n_{1}, n_{2} \geq 4$, consider the induced paths following:

$$
\begin{aligned}
& Q_{1}=\left(w_{21}, w_{11}, w_{2 n_{2}}\right), \\
& Q_{2}=\left(w_{21}, w_{1 n_{2}}, w_{2 n_{2}}\right) \\
& Q_{3}=\left(w_{\left(n_{1}-1\right) 1}, w_{n_{1} 1}, w_{\left(n_{1}-1\right) n_{2}}\right), \\
& Q_{4}=\left(w_{\left(n_{1}-1\right) 1}, w_{n_{1} n_{2}}, w_{\left(n_{1}-1\right) n_{2}}\right), \\
& Q_{5}=\left(w_{12}, w_{21}, w_{31}, \ldots, w_{\left(n_{1}-1\right) 1}, w_{n_{1} 2}\right), \\
& Q_{6}=\left(w_{1\left(n_{2}-1\right)}, w_{2 n_{2}}, w_{3 n_{2}} \ldots, w_{\left(n_{1}-1\right) n_{2}}, w_{n_{1}\left(n_{2}-1\right)}\right), \\
& Q_{i}^{\prime}=\left(w_{i 1}, w_{i 2}, \ldots, w_{i n_{2}}\right) \text { for each } i=1,2 \ldots, n_{1} .
\end{aligned}
$$

Then $\mathcal{S}=\left\{Q_{1}, Q_{2}, \ldots, Q_{6}, Q_{1}^{\prime}, Q_{2}^{\prime} \ldots, Q_{n_{1}}^{\prime}\right\}$ is the collection satisfying the condition mentioned in Remark 7 so that $\eta_{i a}(G)=m-n$, and this completes the proof of the theorem.

Theorem 26. For the graph $G=C_{n_{1}} \circ C_{n_{2}}$ where $n_{1}, n_{2} \geq 3$ and $n_{2} \geq n_{1}$

$$
\eta_{i a}(G)= \begin{cases}m, & \text { if } n_{1}=n_{2}=3 \\ m-n, & \text { otherwise. }\end{cases}
$$

Proof. When $n_{1}=n_{2}=3, G$ is a complete graph on nine vertices, and therefore $\eta_{i a}(G)=m$. Suppose $n_{1} \geq 3$ and $n_{2} \geq$ 4. Consider the induced paths

$$
\begin{aligned}
& Q_{i}=\left(w_{i 2}, w_{i 1}, w_{i n_{2}}\right) \text { for each } i=1,2, \ldots, n_{1}, \\
& Q_{i}^{\prime}=\left(w_{i 2}, w_{i 3}, w_{i n_{2}}\right) \text { for each } i=1,2, \ldots, n_{1}, \\
& Q_{i}^{\prime \prime}=\left(w_{(i+1) 1}, w_{i 2}, w_{(i+1) 3}\right) \\
& \quad \text { for each } i=1,2, \ldots,\left(n_{1}-1\right), \\
& Q_{i}^{\prime \prime \prime}=\left(w_{(i+1)\left(n_{2}-2\right)}, w_{i n_{2}}, w_{(i+1) n_{2}}\right) \\
& \quad \text { for each } i=1,2, \ldots,\left(n_{1}-1\right), \\
& Q^{*}=\left(w_{\left(n_{1}-1\right) 1}, w_{n_{1} 2}, w_{\left(n_{1}-1\right) 3}\right), \\
& Q^{* *}=\left(w_{\left(n_{1}-1\right) 1}, w_{n_{1} n_{2}}, w_{\left(n_{1}-1\right) 3}\right) .
\end{aligned}
$$


Then $\delta=\left\{Q_{1}, Q_{2}, \ldots, Q_{n_{1}}, Q_{1}^{\prime}, Q_{2}^{\prime}, \ldots, Q_{n_{1}}^{\prime}, Q_{1}^{\prime \prime}, Q_{2}^{\prime \prime}, \ldots\right.$, $\left.Q_{\left(n_{1}-1\right)}^{\prime \prime}, Q_{1}^{\prime \prime \prime}, Q_{2}^{\prime \prime \prime}, \ldots, Q_{\left(n_{1}-1\right)}^{\prime \prime \prime}, Q^{*}, Q^{* *}\right\}$ is a collection of induced paths that are edge-disjoint and internally-disjoint such that each vertex of $G$ is an internal vertex of some path in $\mathcal{S}$. Therefore by Remark $7, \eta_{i a}(G)=m-n$.

Remark 27. Since the lexicographic product of two graphs is not commutative, the case when $n_{1}>n_{2}$ is to be considered separately. However, in this case using the same argument followed in Theorems 25 and 26 we get $\eta_{i a}\left(P_{n_{1}} \circ P_{n_{2}}\right)=$ $m-n+4$ when $n_{1}=2$ and $\eta_{i a}\left(P_{n_{1}} \circ P_{n_{2}}\right)=m-n$ otherwise. Also, $\eta_{i a}\left(C_{n_{1}} \circ C_{n_{2}}\right)=m-n$ when $n_{1}>n_{2}$.

Theorem 28. Let $G=P_{n_{1}} \circ C_{n_{2}}$ where $n_{1} \geq 2$ and $n_{2} \geq 3$. Then

$$
\eta_{i a}(G)= \begin{cases}m-n+6, & \text { if } n_{2}=3, \\ m-n, & \text { otherwise. }\end{cases}
$$

Proof. The result follows from Theorem 24 as $P_{n_{1}} \circ C_{3}=P_{n_{1}} \otimes$ $C_{3}$, and all the induced paths considered in Theorem 24 to obtain the collection $\delta$ mentioned in Remark 7 are induced paths in $P_{n_{1}} \circ C_{n_{2}}$ as well.

Remark 29. For the graph $G=C_{n_{1}} \circ P_{n_{2}}$, it can easily be shown that $\eta_{i a}(G)=m$ if $n_{1}=3$ and $n_{2}=2$ and $\eta_{i a}(G)=$ $m-n$ in all other cases.

\section{Scope for Further Research}

Given any graph $G$, the lower bound for $\eta_{i}$ as well as $\eta_{i a}$ is $m-n$. This is because the maximum value that $t_{i}(G)$ and $t_{i a}(G)$ can take is $n$. It has been found in $[10,11]$ that the families of graphs for which this lower bound is attained are very large. In this study on product graphs, we observe that $\eta_{i}$ and $\eta_{i a}$ attain the bound $m-n$ for several product graphs. Therefore, the present study leads us to problems like the following that need further investigation.

(i) Characterize the product graphs for which $\eta_{i}=m-n$.

(ii) Characterize the product graphs for which $\eta_{i a}=m-$ $n$.

(iii) Characterize the product graphs for which $\eta_{i}=\eta_{i a}=$ $m-n$.

\section{Acknowledgment}

The authors would like to thank the two anonymous referees for their constructive and valuable suggestions that played a major role in enriching the content with more results and improving the presentation of the paper.

\section{References}

[1] G. Chartrand and L. Lesniak, Graphs and Digraphs, CRC Press, Boca Raton, Fla, USA, 4th edition, 2005.

[2] F. Harary, "Covering and packing in graphs. I," Annals of the New York Academy of Sciences, vol. 175, pp. 198-205, 1970.
[3] F. Harary and A. J. Schwenk, "Evolution of the path number of a graph: covering and packing in graphs. II," in Graph Theory and Computing, R. C. Rad, Ed., pp. 39-45, Academic Press, New York, NY, USA, 1972.

[4] B. Péroche, "The path-numbers of some multipartite graphs," Annals of Discrete Mathematics, vol. 9, pp. 195-197, 1980.

[5] R. G. Stanton, D. D. Cowan, and L. O. James, "Some results on path numbers," in Proceedings of the Louisiana Conference on Combinatorics, Graph Theory, and Computing, pp. 112-135, 1970.

[6] B. D. Acharya and E. Sampathkumar, "Graphoidal covers and graphoidal covering number of a graph," Indian Journal of Pure and Applied Mathematics, vol. 18, no. 10, pp. 882-890, 1987.

[7] S. Arumugam, "Path covers in graphs," in Lecture Notes of the National Workshop on Decompositions of Graphs and Product Graphs Held at Annamalai University, Tamil Nadu, India, January 2006.

[8] K. Ratan Singh and P. K. Das, "Induced graphoidal covers in a graph," International Journal of Mathematical and Statistical Sciences, vol. 2, no. 3, pp. 102-106, 2010.

[9] K. Ratan Singh and P. K. Das, "Induced acyclic graphoidal covers in a graph," International Journal of Computational and Mathematical Sciences, vol. 4, no. 7, pp. 333-339, 2010.

[10] I. Sahul Hamid and M. Joseph, "Further results on induced graphoidal decomposition," submitted to. Discrete Mathematics, Algorithms and Applications.

[11] I. Sahul Hamid and M. Joseph, "Decomposition of graphs into internally disjoint induced paths," submitted.

[12] R. Hammack, W. Imrich, and S. Klavžar, Handbook of Product Graphs, CRC Press, New York, NY, USA, 2nd edition, 2011. 


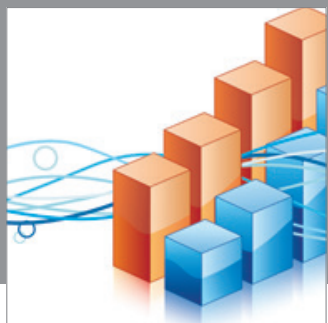

Advances in

Operations Research

mansans

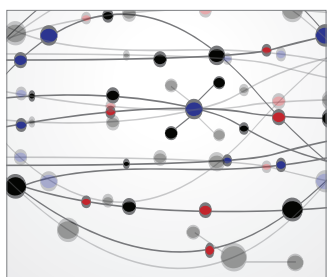

The Scientific World Journal
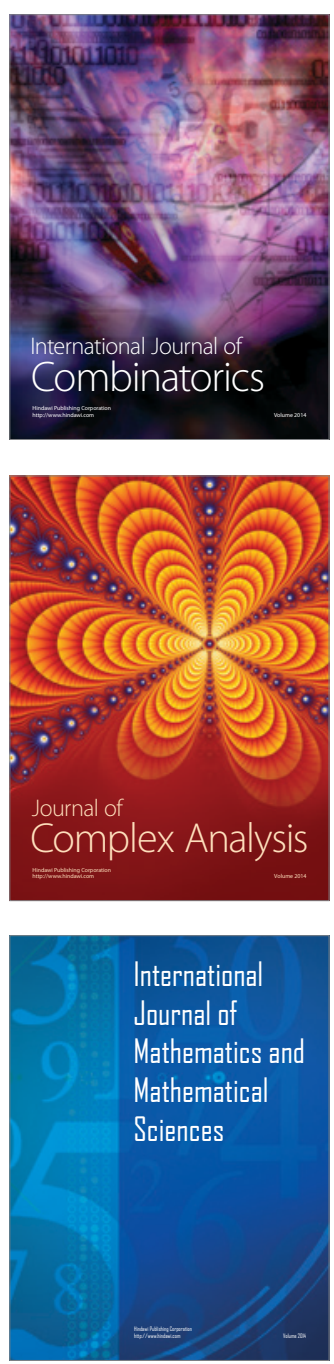
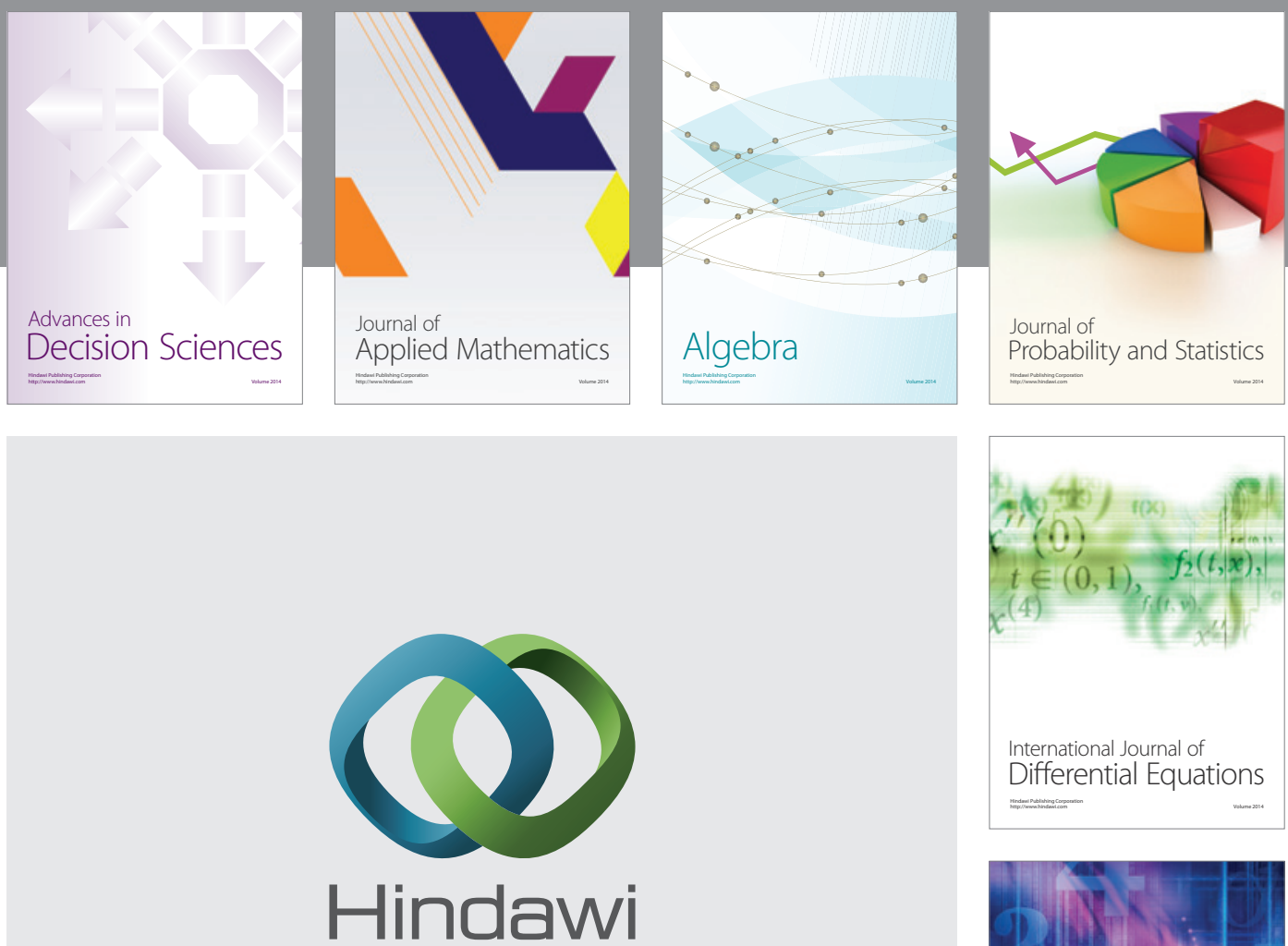

Submit your manuscripts at http://www.hindawi.com
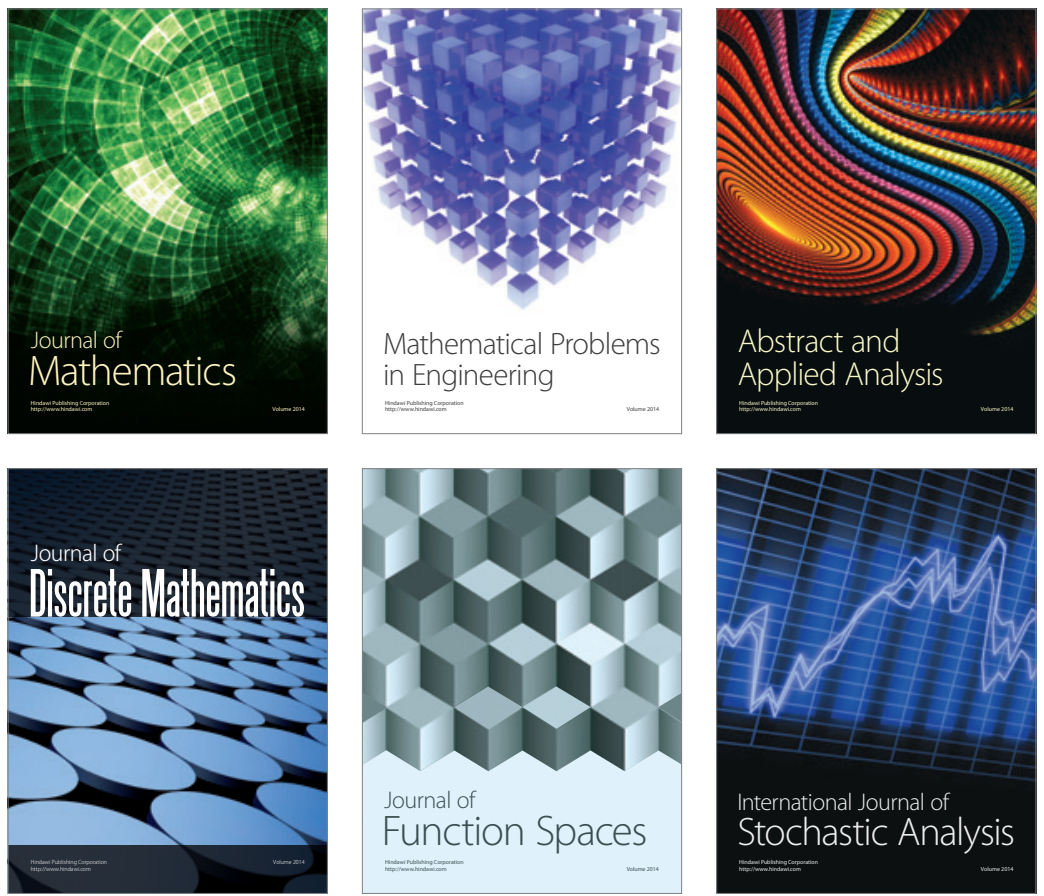

Journal of

Function Spaces

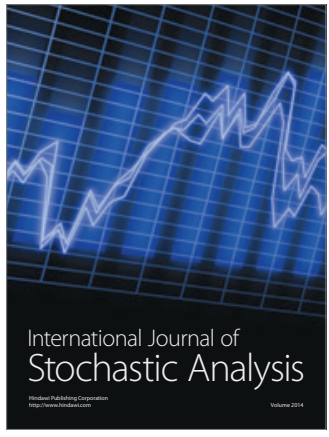

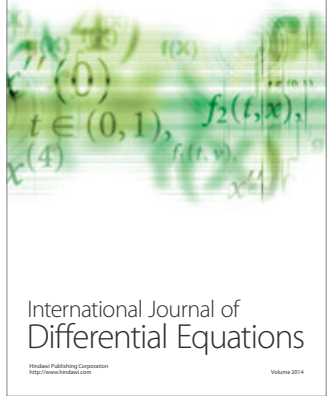
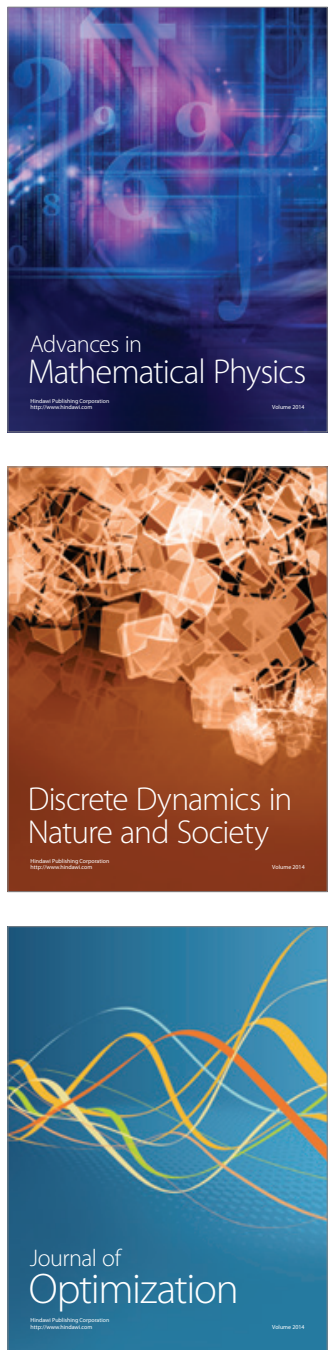